\title{
Facial Reanimation Via 'Free Gracilis Muscle Vascularised Graft'(FGMVG) after Remodelling Resection (RR) in Recurrent Orbitofrontal Fibrous Dysplasia (ROFFD) with Skull Base Extension- Review of Literature
} Tripathy SR*, Mohanta I, Mishra SS and Satapathy MC

Department of Neurosurgery, SCB Medical College, Cuttack, Odisha, India

\begin{abstract}
Background: Fibrous dysplasia (FD) is an abnormal bony lesion of unknown aetiology with insufficient bone maturation and fibrous tissue replacement. It can affect any bone but maxilla is most commonly affected facial bone. Affection of craniofacial bones with skull base involvement, though rare, is important for neurosurgeons. Orbital invasion is very rare. Diagnosis is late due to nonspecific symptoms, low suspicion and relative rarity. Polyostotic FD grows very rapidly and is frequently associated with deformities, complications and recurrence.

Case report: A 35 year old male presented with painful tender swelling, chronic discharging sinus, skin defect and foul smelling discharge over the previous operated site of left fronto - orbital region. He also had 2 episodes of generalised tonic clonic convulsions. He was operated for left frontal FD 15 yrs back. The current CT scan revealed a solid lesion in the left fronto-ethmoido-orbital region extending to skull base

with left frontal lobar compression. He underwent a two stage surgery; first for surgical excision of diseased bone and after 1 month for filling the bony defect with gracillis muscle free vascular graft (FGMVG), anatomising with the superficial temporal vessels. Biopsy confirmed disorganized and immature bone. Patient's symptoms got resolved with improvement of the facial deformity.

Conclusion: Craniofacial fibrous dysplasia involving orbit and skull base is a very rare osteopathy. Re-do surgery may be required even after an interval of one and a half decade. In extensive literature research, ROFFD treated with FGMVG couldn't be traced. So this may be a first such case report; adding to the armamentarium of the Neurosurgeons and craniofacial surgeons in the fight against this dreaded disease.
\end{abstract}

Keywords: Fibrous dysplasia; Cranial base; Facial deformity; Gracilis graft; Discharging sinus

\section{Introduction}

Fibrous dysplasia (FD) is an uncommon benign bony tumour with an unknown aetiology. It entails replacement of the normal bone by a disorganized fibrous tissue containing immature asteroid trabeculae. FD affects mostly individuals of white race during their childhood and adolescence; with a discreet predilection for the female sex. A few rare cases of malignancy have been described, at a rate of $0.5 \%$ to $4 \%$.

Being uncommon and presenting with nonspecific symptoms, it often leads to a late diagnosis for its low level of suspicion. FD has an asymptomatic course because of very slow growth and a tendency for stabilization after puberty [1]. Only in a few cases, FD shows aggressive behaviour and causes anatomic disorders and deformities [2]. Malignant transformation is seen in less than $1 \%$ of the cases.

This case report describes an unusual manifestation of recurrent fibrous dysplasia of fronto-orbital region with extension into skull base characterized by extensive affection of the orbit, paranasal sinus with an aggressive behaviour. In recurrent cases, which is n't uncommon in this disease, free gracilis muscle vascularised graft' (FGMVG) after a second remodelling resection can be a logical step; providing optimal esthetic as well as functional outcome.

In extensive literature research, ROFFD treated with FGMVG couldn't be traced. So this may be a first such case report; adding to the armamentarium of the Neurosurgeons and craniofacial surgeons in the fight against this dreaded disease.

\section{Case Report}

A 35 years old male presented with a painful tender swelling, chronic discharging sinus, a skin defect and foul smelling discharge over the previous operated site of fronto-orbital region. He also had 2 episodes of generalised convulsions. He was operated elsewhere for left fronto-orbital FD in 1998 ((Figure 1A and 1B): the then $\mathrm{x}$ ray skull; (Figure 2): CT brain bone window and (Figure 3): Histopathology). Current (2013) X ray skull (Figure 4) CT scan of brain (Figure 5) and MRI of brain (Figure 6) show a recurrence. His visual acuity was $6 / 6$ on right side and absent perception of light on the left. There was normal olfaction to all types of smell stimuli.

The current CT scan of brain revealed an expansive lesion with osseous density and a ground glass appearance, in the left frontoethmoido-orbital region extending to skull base, compressing the frontal lobe; thereby suggesting recurrent FD involving skull base. $\mathrm{He}$

*Corresponding author: Soubhagya Ranjan Tripathy, Department of Neurosurgery, SCB Medical College, Cuttack, Odisha, India, Tel: 919861008487 ; Fax: 492118104514, E-mail: soubhagya.tripathy@gmail.com

Received September 20, 2015; Accepted October 17, 2015; Published October 20 2015

Citation: Tripathy SR, Mohanta I, Mishra SS, Satapathy MC (2015) Facial Reanimation Via 'Free Gracilis Muscle Vascularised Graft' (FGMVG) after Remodelling Resection (RR) in Recurrent Orbitofrontal Fibrous Dysplasia (ROFFD) with Skull Base Extension- Review of Literature. J Neurol Disord S1: 003. doi:10.4172/2329-6895.S1-003

Copyright: (c) 2015 Tripathy SR, et al. This is an open-access article distributed under the terms of the Creative Commons Attribution License, which permits unrestricted use, distribution, and reproduction in any medium, provided the original author and source are credited. 
Citation: Tripathy SR, Mohanta I, Mishra SS, Satapathy MC (2015) Facial Reanimation Via 'Free Gracilis Muscle Vascularised Graft' (FGMVG) after Remodelling Resection (RR) in Recurrent Orbitofrontal Fibrous Dysplasia (ROFFD) with Skull Base Extension- Review of Literature. J Neurol Disord S1: 003. doi:10.4172/2329-6895.S1-003

underwent a two staged operation. $1^{\text {st }}$ - surgical excision of diseased bone (The post op CT scan of brain ((Figure 7): Axial, (Figure 8): Sagittal: show a fair resection. (Figure 9): The patient before reconstruction) and
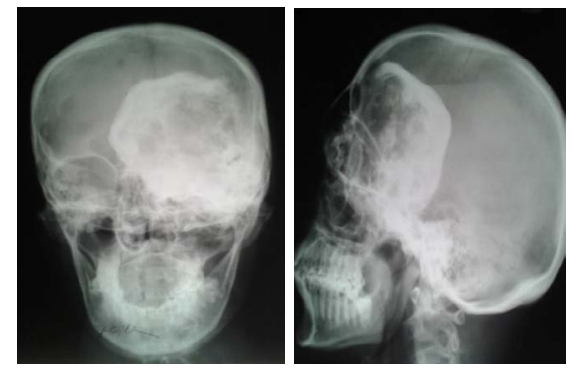

Figure 1: Plain X ray of skull (Lateral view) in 1998, showing a fibrous dysplasia with ground glass appearance destroying the orbital cavity, frontal bone with intracranial extension.

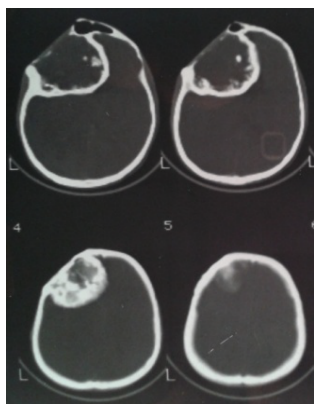

Figure 2: Computed tomography bone window view (1998), confirming an expansive lesion with osseous density and ground glass appearance involving the frontal bone with intracranial extension.

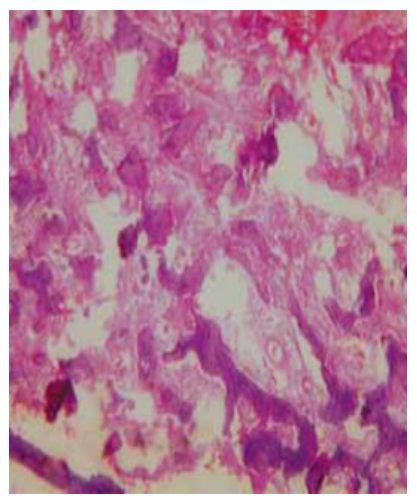

Figure 3: Histopathological report showing the normal bone replaced by fibrous tissue with immature and disorganized trabeculae.

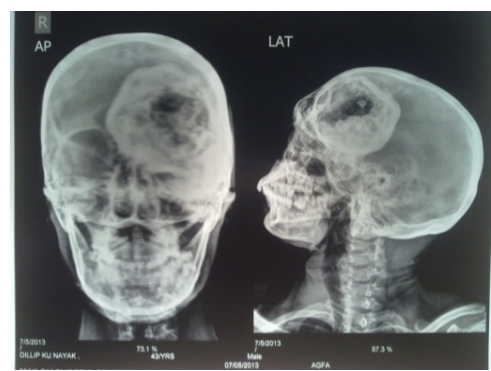

Figure 4: Plain Skull X ray (AP and Lateral view) in 2013, showing a recurrent fibrous dysplasia at the left fronto-orbital bone with intracranial extension to skull base.

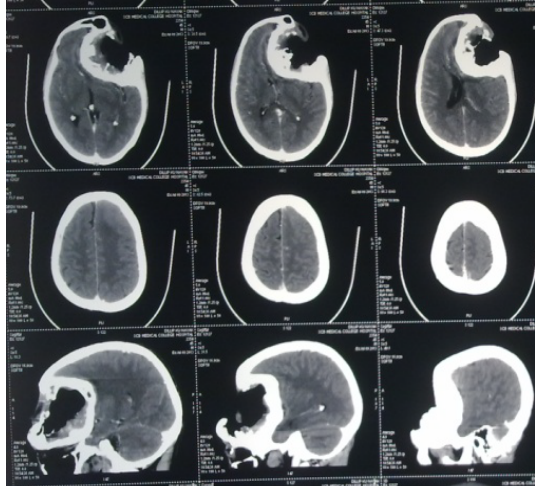

Figure 5: Computed tomography sagittal view in 2013, confirming an expansive lesion with osseous density involving the frontal and sphenoidal bone with extension to skull base.

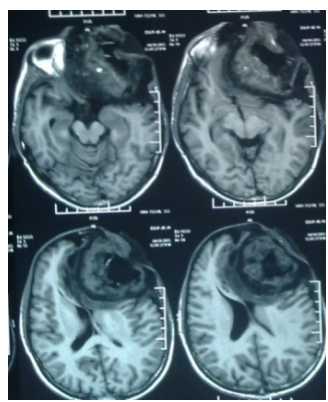

Figure 6: MRI of brain axial view (2013), confirming an irregular expansive lesion with mass effect and perilesional edema involving the left frontal lobe with extending upto midline structures.

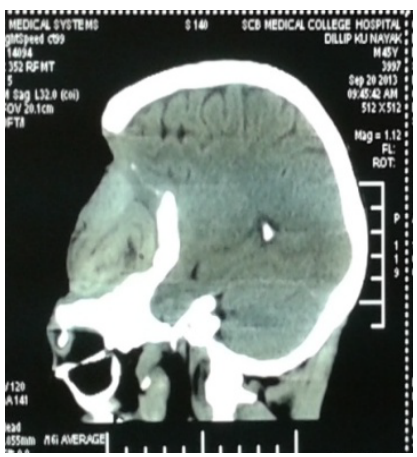

Figure 7: Post op sagittal CT scan of brain, showing subtotal resection of the diseased bone with a small residual attached to sphenoid bone.

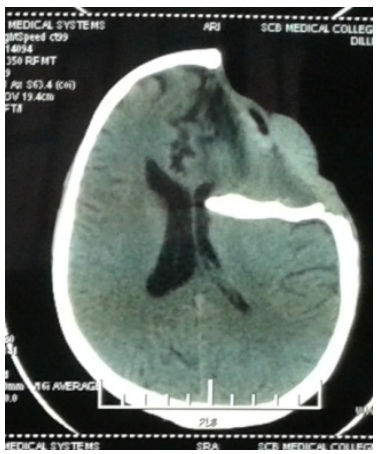

Figure 8: Post op axial CT scan of brain, showing subtotal resection of the diseased bone with a small residual and post operative gliotic changes in brain. 
Citation: Tripathy SR, Mohanta I, Mishra SS, Satapathy MC (2015) Facial Reanimation Via 'Free Gracilis Muscle Vascularised Graft' (FGMVG) after Remodelling Resection (RR) in Recurrent Orbitofrontal Fibrous Dysplasia (ROFFD) with Skull Base Extension- Review of Literature. J Neurol Disord S1: 003. doi:10.4172/2329-6895.S1-003

Page 3 of 6

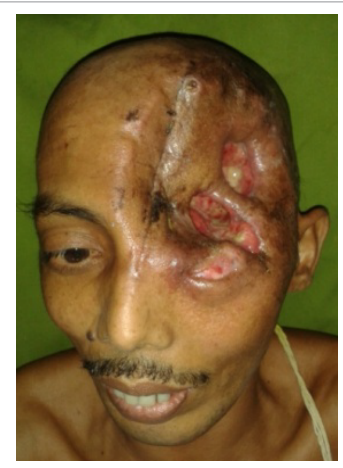

Figure 9: Photograph of patient after subtotal excision of the dysplastic bone with residual cavity with granulation tissue formation.

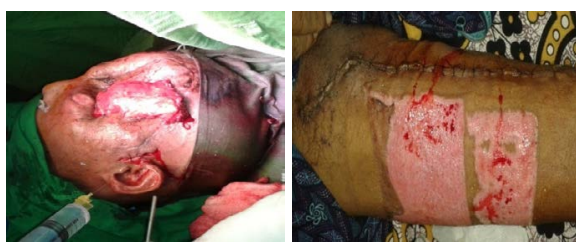

Figure 10: Intra operative photograph of patient showing residual cavity filled with gracilis muscle free vascular pedicle graft anastomosed with left superficia temporal vessel.

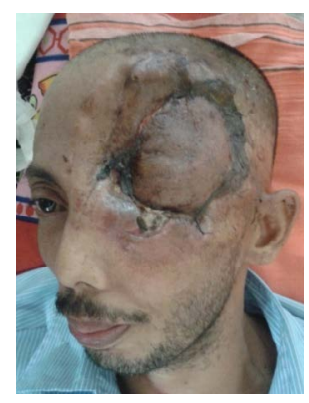

Figure 11: Postoperative photo of patient showing cavity filled with gracilis muscle graft and split skin graft.

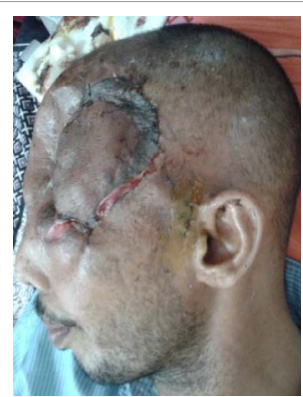

Figure 12: Postoperative photo of patient showing cavity filled with gracilis muscle graft and split skin graft.

after 1 month, filling the bony defect was done with gracillis muscle free vascular graft, having anastomosis with the superficial temporal vessels (Figure 10).

Histopathological examination confirmed normal bone replaced with fibrous tissue, containing disorganized immature bone. There was gradual resolution of symptoms with improvement of the facial deformity. The vision and olfaction were preserved post operatively.
The patient has gone back to his regular activity (Figures 11 and 12) and was doing well on his last visit $2 \frac{1}{2} 2$ years after the surgery.

\section{Discussion}

Von Recklinghausen first described the condition in a patient with skeletal deformities and coined the term "osteitis fibrosa generalisata". It was renamed by Bauer as "osteitis fibrosa" [3] and "fibrous dysplasia" by Lichtenstein [4]. Schlumberger first reported single bone involvement by the disease process and described it as "monostotic fibrous dysplasia" [5]. Craniomaxillofacial "(CMF) fibrous dysplasia" was first reported by Thoma et al. [6]. Reed has defined the condition as an "arrest of bone maturation in woven bone with ossification resulting from metaplasia of a nonspecific fibro-osseous type" [7].

Monostotic fibrous dysplasia (MFD), Polyostotic fibrous dysplasia (PFD), and McCune- Albright Syndrome (MAS) belong to a spectrum of substitution of the normal osseous maturation by a disorganized fibrous tissue that contains immature asteroid trabeculae, similar to 'Chinese letters'. MFD and PFD are characterized by fibrodysplastic bone of one or more skeletal sites. MAS has been classically described as a triad of precocious puberty, cafe-au-lait spots, and PFD. The present day definition has been broadened to not only include precocious puberty, but other hyper-functioning endocrinopathies as well.

The genetic aetiopathogenesis of these 3 disorders constitutes a somatic mutation of the GNAS gene on chromosome 20q13, leading to impaired GTPase activity in the protein Gsa, with altered cAMP signalling; resulting in increased proliferation of the cells of the osteogenic lineage in the bone marrow. The spectrum of the phenotypic severity depends on the migration and survival of the mutated cells during embryonic development. While PFD and MAS are relatively uncommon entities, craniofacial fibrous dysplasia is frequently found in these patients and has been reported in the literature to occur in up to $50-100 \%$ of cases [8].

The most commonly involved bones are femur, tibia, ribs and facial bones. Craniofacial FD represents $2.5 \%$ of the osseous tumours and $7.5 \%$ of benign tumours of bone. White population and female gender are mostly affected. It is generally believed that the condition is more frequent in children and does not increase after the period of bone growth i.e. adolescence. Monostotic FD, generally presents an asymptomatic or slight symptomatic course, with insidious growth and tendency for stabilization after puberty. The discovery is usually incidental. Only in a few cases it has a more aggressive growth and provokes pain, nasal obstruction and exophthalmia. Polyostotic FD is associated with a progressive affection, high chances of complications and recurrences at the adult age. Orbital pain, proptosis, nasal obstruction, and headache- are nonspecific, insidious pictures that evolve with other aesthetic alterations, such as convexity of the nasal pyramid and ocular proptosis. The main symptoms are sensorineural dysacusis, stenosis of the auditory meatus, unilateral and bilateral nasal obstruction, intense headache, exophthalmia. The malignant transformation is observed in less than $1 \%$ of the cases.

The craniofacial involvement in monostotic type is $25 \%$ and in polyostotic type is $50 \%$. In face, the maxilla is most commonly affected bone followed by the jaw. The frontal, temporal and clivus bones are less affected. The extension to the orbit is rare, and the cranial base invasion is a very uncommon event [9].

Visual dysfunction and optic atrophy occurs in the lesions involving the sphenoid wing, encroaching upon the optic canal [10]. Typically, the visual loss is slowly progressive, which could be unilateral or even bilateral. Infrequently, visual deterioration could be rapid, ranging 
Citation: Tripathy SR, Mohanta I, Mishra SS, Satapathy MC (2015) Facial Reanimation Via 'Free Gracilis Muscle Vascularised Graft' (FGMVG) after Remodelling Resection (RR) in Recurrent Orbitofrontal Fibrous Dysplasia (ROFFD) with Skull Base Extension- Review of Literature. J Neurol Disord S1: 003. doi:10.4172/2329-6895.S1-003

Page 4 of 6

from few hours to a few days. Acute visual loss is probably the result of the vascular event, affecting the ophthalmic artery. Acute visual deterioration can also result from associated pathologies like mucocele or aneurysmal bone cyst around the vicinity of the optic canal. Visual improvement has been reported in 7 of the 9 adult patients with acute visual loss in one study [11]. Optic canal decompression was recommended as the procedure of choice in patients with mild visual deterioration due to fibrous dysplasia, despite the elevated hemorrhage risk, taking into account the individual risk/benefit ratio with the aim to improve the quality of life.

The following radiologic forms are described [12]:

(1) Compact form- M/C (50\%), a homogeneous hyperdensity with "ground glass"appearance.

(2) Lytic lesion- an uneven nature surrounded by hyperdensity margin.

(3) Mixed form- radiopaque areas with radiolucent areas.

The main differential diagnoses are: Hyperostosis, Osteoma, Osteosarcoma, Chordoma, Hyperostotic meningioma, Brown tumour of the hyperthyroidism and Ossifying fibroma.

CT signs comprise bone hypertrophy straddling cranial sutures, with conserved bone concavity. Diagnosis is indicated by a juxtaposition of "frosted glass" condensation areas and areas of demineralization. On MRI, the lesion presents as variable iso signal on T1-weighted images, hyper-signal on T2 with heterogeneous gadolinium uptake.

Surgical treatment is indicated for symptomatic lesions and may be done at any age. Sometimes it is not possible to perform complete excision of the tumour due to the degree of affection, nondesigning of tumoral margins and critical location of the tumor. Fibrous dysplasia usually does not respond to radiotherapy and is rather considered to be a factor for malignant transformation. The aim of the treatment is to correct cosmetic deformity incurred due to overgrowth of bone or decompression of the neural structures producing symptoms. In adults, visual deterioration due to fibrous dysplasia is usually a slow process and occurs over months or years. Optic nerve decompression is a logical option in patients with visual loss.

Remodelling resection is the reference technique. The coronal approach is a good primary option for optic nerve decompression. The principles of fibrous dysplasia surgery are maximal tumor tissue resection, functional restoration, and esthetic optimization. Reconstructions are favourable in case of anatomic distortions, functional alterations and compression of cranial nerves, and usually indicated after stabilization of the tumoral growth and must be immediately performed after a large resection of the osseous lesions with the autologous bone grafts or polymethyl methacrylate.

Reconstructive surgery for defects resulting from head and neck cancer allows tumor resection while maintaining quality of life. Various treatment procedures involving fat grafts and local or microvascular free flaps have been used for correction of facial contour deformities. Free flaps have been in clinical use for nearly three decades. In that time, their survival rates have improved as refinements have been made in surgical technique and instrumentation. ROFFD do cause externally visible deformities.

There have been various attempts to surgically manage fibrous dysplasia especially in facial bones. Artificial implants like silicone or hydroxyapatite were used to correct the defect on the face. Autologous fat injection or dermofat graft, bone graft are also commonly used surgical methods. Recently, perforator-based ALT dermofat flap (anterolateral thigh free flap) is being widely applied to correct the asymmetry of the face [13].

Microvascular tissue transfer, this technique began to be used to cover defects in the oral and maxillofacial area in the early 1980's. Currently, many different types of flap are used, with varying characteristics. Each type of flap has a spectrum of indications that may be broad or narrow; for special types of defect, multiple flaps can be used. Finding the optimal solution to a given reconstructive problem involves a determination of the most suitable tissue, the most favourable donor site, and the physiologically least stressful operation for the patient [14]. Because this method leaves the superfluous fatty and muscular tissue at the donor site rather than taking it along with the flap, a very thin flap results that is highly suitable for coverage of the usually flat defects of the oral cavity or facial skin [15].

Mao et al, analyze the feasibility and reliability of free tissue flap transfers in the head and neck region. One thousand five hundred and eighty-four consecutive free flap transfers performed in 1,501 patients were reviewed. There were ten kinds of free flaps in this group, with the free fibula flap most commonly used, followed by free radial forearm flap, rectus abdominis flap, free jejunum flap, anterolateral thigh free flap, iliac crest flap, scapular flap, latissimus dorsi flap, lateral arm flap, and ileocecal flap. The overall success rate of flaps was $98.1 \%$ $(1,554 / 1,584)[16]$.

In particular order, patient had done free tissue flap transfers to fill the mandible and maxilla defect with free fibula flap, free radial form arm flap, and at last, anterolateral thigh free flap. It indicates that multiple stage reconstruction must be done. The ALT flap, first described by Song et al, has gained popularity in head and neck reconstructive surgery. The ALT flap is a versatile soft-tissue flap in which thickness and volume can be adjusted for the extent of the defect. In our experience, flap elevation is relatively easy, although surgeon should be familiarized with the anatomy of donor site. It has a consistent anatomy of the main pedicle (descending branch of the lateral femoral circumflex vessels).

The ALT and the proximal lateral calf have been described as particularly suitable donor sites for reconstruction in oral and maxillofacial surgery. The widest experience to date has been with ALT perforator-based flaps up to $8 \times 25 \mathrm{~cm}$ in size, which are currently used routinely by some authors as an alternative to radial flaps because of the very low morbidity at the donor site, and which have a wide spectrum of indications. In contrast, Soleus perforator-based flaps are usually used only for smaller defects, because the donor site in the proximal lateral thigh can only be primarily closed if the flap is $5 \mathrm{~cm}$ wide or less. Preservation of the cutaneous vessels of the calf keeps the morbidity of flap harvesting to a minimum. By deepithelizing the ALT flap, as dermofat flap, no loss in the bulk of the flap and flap perfusion is observed. The dermofat flap allows easy creation of a subcutaneous pocket under the cheek through the available upper incision line without extra scarring. The major advantages of the ALT dermofat flap were as follows: provision of a constant and safe pedicle, adequate bulk, a low risk of atrophy and infection by means of vascularized tissue, and well-hidden scar at the donor site.

The controversies regarding the timing, indications, amount of resection, and orbital decompression in craniofacial dysplasia have been addressed below for a comprehensive treatment strategy.

Surgery can wait for the end of the growth phase (i.e., adolescence) and the patients should be counselled regarding recurrence and absence of any significant correlation between the sites of occurrence to 
Citation: Tripathy SR, Mohanta I, Mishra SS, Satapathy MC (2015) Facial Reanimation Via 'Free Gracilis Muscle Vascularised Graft' (FGMVG) after Remodelling Resection (RR) in Recurrent Orbitofrontal Fibrous Dysplasia (ROFFD) with Skull Base Extension- Review of Literature. J Neurol Disord S1: 003. doi:10.4172/2329-6895.S1-003

Page 5 of 6

the rapidity of progress. Functional status dictates over esthetic issues in the decision making process.

Chen and Noordhoff proposed a treatment algorithm for the management of craniomaxillofacial fibrous dysplasia incorporating aggressive, radical surgery for the resection of diseased tissue [17]. For this algorithm, they proposed that the head and face could be divided into 4 zones based on the esthetic and functional consequences of the disease at each of these sites and the unique anatomic considerations for operating in each area.

Zone 1 represents the fronto-orbito-malar regions of the face. These are esthetically critical and can be adequately reconstructed with simple bone grafting techniques after reconstruction. For this region, they recommended radical excision and reconstruction.

Zone 2 refers to the hair bearing scalp. It is not typically an aesthetic concern, and as such, intervention is optional for the patient.

Zone 3 refers to the central skull base including the sphenoid, pterygoid, petrous temporal bone, and mastoid. Given the difficulty in obtaining surgical access to these areas, the authors recommended observation of lesions in this region.

Zone 4 comprises the tooth bearing portions of the skull, the maxilla and mandible. The authors recommended conservative management, given the difficulty in reconstructing defects in this region.

Medical therapy has not occupied a prominent role in the management of fibrous dysplasia to date. Recognition of pathogenesis of this disease led to treatment with biphosphonates. They control bone erosion by the inhibition of osteoclastic action. They have a high affinity for hydroxyapatite of resorbed bone and remain tied to it for a long period. The drug is incorporated into the cellular cytoplasm, inhibiting acid phosphatase secretion thereby arresting bone resorption [18]. The theory is that the drug stabilizes the disease and improves the patient's pain. Another drug that has been used is pamidronate given intravenously. Pamidronate containing a basic nitrogen atom in its alkyl side chain represents a second generation drug, characterized by increased potency of inhibition of bone resorption and good tolerance [19].

Bisphosphonate (pamidronate $60 \mathrm{mg} / \mathrm{d}$ ) injection for 3 consecutive days every 6 months for 18 months can be tried in case of pain; alleviating pain and reducing bone resorption in $50 \%$ of cases. No mandibular osteonecrosis was seen as a result. The authors recommended stopping treatment 3 months ahead of scheduled surgery [20]. In the described case, the patient was not administered this regimen. Vitamin D (800 IU/ day) and calcium supplementation $(1 \mathrm{~g} /$ day) are also recommended due to low serum levels [21]. Radiation therapy should not be applied in fibrous dysplasia, which can induce a 400-fold elevation in malignancy risk [22].

Prophylactic orbital decompression is controversial. Increased intraluminal pressure in the retinal vein due to external compression of the optic canal inducing arterial steal explains the compressive mechanism; also the optic nerve canal may be compressed by a cyst. $95 \%$ of patients conserve normal vision despite optic canal stenosis. Some authors have suggested prophylactic decompression [21]. Regular visual assessment should be undertaken when stenosis is diagnosed; decompression should be embarked upon only in cases of continuously deteriorating visual acuity. It arrests deterioration in more than half of cases, usually with actual improvement [16]. Prophylactic decompression should not be performed in first intention, as impairment is found during the following year in a third of cases.

The risk of recurrence being high, at $25 \%$, functional impact should take precedence over esthetic considerations in guiding therapy. In a large series $22 \%$ had polyostotic and $76 \%$ monostotic involvement. 61 of the 68 patients managed by surgery had radical lesion resection; 6 received conservative treatment; one had 1-step mandibular resection with free peroneal flap reconstruction. There were no cases of recurrence following radical resection, and the author therefore recommended this radical but definitive solution in involuted monostotic forms [18]. In contrast, Choi demonstrated the interest of a non-invasive attitude, with a minimal vestibular or subciliary approach depending on the lesion, in which radical resection is precluded by skull-base involvement [23-25].

Recently developed dedicated endonasal surgical instrumentation with curved forceps, angulate dreamers and neuronavigation, enhances surgical precision. Advances in imaging, connecting a $3 \mathrm{dMD}$ surface-imaging camera up to a cone beam computed tomography (CBCT) system, allows evolution to be visualized by fusion of skin and bone images [20].

In a recent article [26] evaluating the surgical options for facial paralysis, covering primary neurorrhaphy to facial reanimation, with microvascular free tissue transfer; the summary was: "although primary facial nerve repair attains the best results, nerve grafting with the sural nerve and commercially available motor nerve allografts can be used alone, or in combination with masseteric nerve grafts to attain facial tone and protect eyelid function. The workhorse for reanimation is the gracilis free tissue transfer innervated by the masseteric nerve or contralateral facial nerve using a cross-face nerve graft. The orthodromic temporalis tendon transfer has minimal donor site morbidity and acceptable reported outcomes".

These encouraging results hold promise for the future of reconstruction of recurrent orbitofrontal fibrous dysplasia (ROFFD) surgery for neurosurgeons as well as craniofacial surgeons worldwide.

\section{Conclusion}

Recurrent orbitofrontal fibrous dysplasia (ROFFD) with skull base extension is a rarity in the rather uncommon benign bone tumour fibrous dysplasia. Remodelling resection (RR) is the index operation. In recurrent cases, which is n't uncommon in this disease, free gracilis muscle vascularised graft' (FGMVG) after a second remodelling resection can be a logical step; providing optimal esthetic as well as functional outcome. In extensive literature research, ROFFD treated with FGMVG couldn't be traced. So this may be a first such case report; adding to the armamentarium of the Neurosurgeons and craniofacial surgeons in the fight against the dreaded disease.

\section{Patient Consent}

The patient /next of kin/guardian has consented to the submission of the case report for submission to the journal.

\section{Competing Interests}

We have no financial/ non-financial (political, personal, religious, ideological, academic, intellectual, commercial or any other) competing interests.

\section{Author's Contribution}

SRT, IM carried out the investigation, data keeping, SRT drafted the manuscript, SRT, IM, MCS helped in preparation of the patient preoperatively and followed up the patient postoperatively; also helped in review of literature, SSM, IM, MCS participated in the surgical procedure, SRT participated in the design of the study and reviewed the literature, SRT, SSM conceived of the study, and participated in its design, coordinated and drafted the manuscript, All authors read and approved the final manuscript. 
Citation: Tripathy SR, Mohanta I, Mishra SS, Satapathy MC (2015) Facial Reanimation Via 'Free Gracilis Muscle Vascularised Graft' (FGMVG) after Remodelling Resection (RR) in Recurrent Orbitofrontal Fibrous Dysplasia (ROFFD) with Skull Base Extension- Review of Literature. J Neurol Disord S1: 003. doi:10.4172/2329-6895.S1-003

\section{References}

1. Alves AL, Canavarros F, Vilela DSA, Granato L, Próspero JD (2002) Displasia fibrosa: relato de três casos. Rev Bras Otorrinolaringol 68: 288-292.

2. Bauer W, Albright F, Aub JC (1930) A case of osteitis fibrosa cystica (osteomalacia?) With evidence of hyperactivity of the para-thyroid bodies. Metabolic study II. J Clin Invest 8: 229-248.

3. Chapurlat RD (2006) Medical therapy in adults with fibrous dysplasia of bone. J Bone Miner Res 21 Suppl 2: P114-119.

4. Chen YR, Noordhoff MS (1990) Treatment of craniomaxillofacial fibrous dysplasia: how early and how extensive? Plast Reconstr Surg 86: 835-842.

5. Choi JW, Lee SW, Koh KS (2009) Correction of proptosis and zygomaticomaxillary asymmetry using orbital wall decompression and zygoma reduction in craniofacial fibrous dysplasia. J Craniofac Surg 20: 326-330.

6. Edgerton MT, Persing JA, Jane JA (1985) The surgical treatment of fibrous dysplasia. With emphasis on recent contributions from cranio-maxillo-facial surgery. Ann Surg 202: 459-479.

7. Hölzle F, Mohr C, Wolff KD (2008) Review Article: Reconstructive Oral and Maxillofacial Surgery. Deutsches Ärzteblatt International. Dtsch Arztebl Int 105: 815-822.

8. Harris BN, Tollefson TT (2015) Facial reanimation: evolving from static procedures to free tissue transfer in head and neck surgery. Curr Opin Otolaryngol Head Neck Surg 23: 399-406.

9. Horgan MA, Delashaw JB, Dailey RA (1999) Bilateral proptosis: an unusual presentation of fibrous dysplasia. Br J Neurosurg 13: 335-337.

10. Kos M, Luczak K, Godzinski J, Klempous J (2004) Treatment of monostotic fibrous dysplasia with pamidronate. J Craniomaxillofac Surg 32: 10-15.

11. Kruse A, Pieles U, Riener MO, Zunker Ch, Bredell MG, et al. (2009) Craniomaxillofacial fibrous dysplasia: a 10-year database 1996-2006. Br J Oral Maxillofac Surg 47: 302-305.

12. Kusano T, Hirabayashi S, Eguchi T, Sugawara $Y(2009)$ Treatment strategies for fibrous dysplasia. J Craniofac Surg 20: 768-770.

13. Lichtenstein L (1938) Polyostotic Fibrous Dysplasia. Archives of Surgery, 36 874-898. http://dx.doi.org/10.1001/archsurg.1938.01190230153012.
14. Lupescu I, Hermier M, Georgescu SA, Froment JC (2001) Helical CT and diagnostic evaluation of cranio-facial fibrous dysplasia. J Radiol 82: 145-149.

15. Mao C, Yu GY, Peng X (2008) Department of Oral and Maxillofacial Surgery, Peking University School and Hospital of Stomatology, Beijing, China. Free composite flap transfers in the head and neck region: an 8-year experience 40: 64-67.

16. Lee JS, FitzGibbon E, Butman JA, Dufresne CR, Kushner H, et al. (2002) Normal vision despite narrowing of the optic canal in fibrous dysplasia. N Eng J Med 347: 1670-1676

17. Reed RJ (1963) Fibrous dysplasia of bone. A review of 25 cases. Arch Pathol 75: $480-495$

18. Ricalde P, Horswell BB (2001) Craniofacial fibrous dysplasia of the fronto-orbita region: a case series and literature review. J Oral Maxillofac Surg 59: 157-167.

19. Schlumberger HG (1946) Fibrous dysplasia of single bones (monostotic fibrous dysplasia). Mil Surg 99: 504-527.

20. Tan YC, Yu CC, Chang CN, Ma L, Chen YR (2007) Optic nerve compression in craniofacial fibrous dysplasia: the role and indications for decompression. Plas Reconstr Surg 120: 1957-1962.

21. Thoma KH (1956) Differential diagnosis of fibrous dysplasia and fibro-osseous neoplastic lesions of the jaws and their treatment. J Oral Surg (Chic) 14: 185-194.

22. Thoma A, Sprague S (2005) Methodologic issues in the comparison of microsurgical flaps/techniques in head and neck reconstruction. Clin Plast Surg 32: 347-359, vi.

23. Valentini V, Cassoni A, Marianetti TM, Terenzi V, Fadda MT, et al. (2009) Craniomaxillofacial fibrous dysplasia: conservative treatment or radical surgery? A retrospective study on 68 patients. Plast Reconstr Surg 123: 653660.

24. Vasikaran SD (2001) Bisphosphonates: an overview with special reference to alendronate. Ann Clin Biochem 38: 608-623.

25. Weischer T, Schettler D, Mohr C (1997) Titanium implants in the zygoma as retaining elements after hemimaxillectomy. Int J Oral Maxillofac Implants 12 211-214.

26. Weisman JS, Hepler RS, Vinters HV (1990) Reversible visual loss caused by fibrous dysplasia. Am J Ophthalmol 110: 244-249.
This article was originally published in a special issue, Management of Neurosurgical Complications handled by Editor(s). Dr. Athanasios K. Petridis, Department of Neurosurgery, University of Schleswig Holstein, Germany

Citation: Tripathy SR, Mohanta I, Mishra SS, Satapathy MC (2015) Facia Reanimation Via 'Free Gracilis Muscle Vascularised Graft' (FGMVG) after Remodelling Resection (RR) in Recurrent Orbitofrontal Fibrous Dysplasia (ROFFD) with Skull Base Extension- Review of Literature. J Neurol Disord S1: 003. doi:10.4172/2329-6895.S1-003
Submit your next manuscript and get advantages of OMICS Group submissions

Unique features:

- Increased global visibility of articles through worldwide distribution and indexing

- Showcasing recent research output in a timely and updated manner

Special issues on the current trends of scientific research

Special features:

- 700 Open Access Journals

50,000 editorial team

Rapid review process

Quality and quick editorial, review and publication processing

- Indexing at PubMed (partial), Scopus, DOAJ, EBSCO, Index Copernicus and Google Scholar etc

Sharing Option: Social Networking Enabled

- Authors, Reviewers and Editors rewarded with online Scientific Credits

Better discount for your subsequent articles

Submit your manuscript at: http://scholarscentral.com/ 